\section{Determining SNP Allele Frequencies in DNA Pools}

\author{
BioTechniques 28:464-470 (March 2000)
}

\section{ABSTRACT}

Single nucleotide polymorphisms (SNPS) are among the most common types of polymorphism used for genetic association studies. A method to allow the accurate quantitation of their allele frequencies from DNA pools would both increase throughput and decrease costs for large-scale genotyping. However, to date, most DNA pooling studies have concentrated on the use of microsatellite polymorphisms. In the case of SNPs that are restriction fragment length polymorphisms (RFLPs), studies have tended to use methods for the quantitation of allele frequency from pools that rely on densitometric evaluation of bands on an autoradiograph. Radiation-based methods have well-known drawbacks, and we present two alternate methods for the determination of SNP allele frequencies. For RFLPs, we used agarose gel electrophoresis of digested PCR products with ethidium bromide staining combined with densitometric analysis of gel images on a PC. For all types of SNP, we used allele-specific fluorescent probes in the Taqman ${ }^{\circledR}$ assay to determine the relative frequencies of two different alleles. Both methods gave accurate and reproducible results, suggesting they are suitable for use in DNA pooling experiments.

\section{INTRODUCTION}

One method to find genes that influence diseases or traits in any organism is by association studies. This involves finding the allele frequencies of genes from a population of organisms with that trait or disease and comparing them to a control population that does not exhibit the disease or trait. In humans, this means examining a disease population and a control or normal population. Large-scale association studies are necessary to detect loci that have only a weak effect on disease susceptibility (7).
This often means laborious and expensive individual genotyping of substantial sets of samples. One approach designed to reduce the time and cost involved is DNA pooling, where equal amounts of DNA from many individuals are mixed together and genotyped. The upper limit on DNA pool size is very large. Some simple calculations reveal that since there are approximately 1200 copies of the human genome in 1 ng of DNA, then using $25 \mathrm{ng}$ of DNA in a PCR would still give an average of 30 complete copies of each person's DNA in a pool of 1000 individuals.

The results from genotyping the pools are interpreted as a representation of the allele frequency distribution in the individual samples. Methods for the accurate quantitation of allele frequencies from DNA pools potentially allow the screening of many candidate polymorphisms in the time it would take to genotype one polymorphism individually. Such methods exist for microsatellites (2-4) but similar methods have not been developed to the same extent for the rapid assessment of single nucleotide polymorphisms (SNPs). What have been published are methods using radioactivity for the assessment of restriction fragment length polymorphisms (RFLPs) (8). We have developed two methods without the drawbacks of using radioactivity. One is densitometric analysis of an RFLP visualized on an agarose gel stained with ethidium bromide; the other relies on the use of allele-specific fluorescent probes in the Taqman ${ }^{\circledR}$ assay (PE Biosystems).

\section{MATERIALS AND METHODS}

\section{Pool Construction}

An equal amount $(1 \mu \mathrm{g})$ of DNA from 50 unrelated human controls was combined together into a DNA pool, which was then diluted to $50 \mathrm{ng} / \mu \mathrm{L}$. Concentrations of samples were estimated by fluorimetry. The fluorimeter used was the DyNAQuant 200 (Amersham Pharmacia Biotech, Herts, UK). A DNA-specific dye (H33258) was also used with an excitation wavelength of $480 \mathrm{~nm}$ and an emission wavelength of $565 \mathrm{~nm}$. The measurements were performed using a $1 \mathrm{~cm}$ mirrored glass cu- vette after calibration with calf thymus DNA standard (Sigma, St. Louis, MO, USA). All samples were measured in duplicate. If the duplicates were more than 5\% different, the sample was remixed and measured again.

\section{Testing the Sensitivity of the DNA Pooling Method}

We tested the accuracy of our pooling protocol by comparing observed allele frequencies with those derived from individual genotyping and then tested its sensitivity by a spiking strategy where we introduced additional alleles to DNA pools. The samples had been individually genotyped for the two markers studied. The pools were then divided into aliquots, and the amount of DNA from a homozygote that was equivalent to one, two, five and ten alleles was added to these aliquots. For example, in a $50 \mu \mathrm{L}$ aliquot, if there were $50 \mathrm{ng}$ from each individual, then the addition of $25 \mathrm{ng}$ of DNA should represent the addition of one allele's worth of DNA, $250 \mathrm{ng}$ ten alleles' worth and so on. PCR was then carried out for pools containing no extra, five extra and ten extra alleles of a specific type. The analysis of the PCR products by two different methods is detailed below.

For the RFLP method, additional calibration pools were constructed. DNA from different homozygotes was mixed together in different ratios $(0: 100,20: 80$, 40:60, etc.) as shown in Figure 2.

\section{Markers}

The SNPs studied were the -141 Del/Ins polymorphism in the human dopamine receptor 2 (DRD2) gene (1) and $2041 \mathrm{G} \rightarrow \mathrm{T}$ in the human collagen $\alpha 1$ (i) or COLIA1 gene (5).

\section{Genotyping}

RFLP Assay: DRD2 -141 Del/Ins. PCR was carried out using primers D2677 and D2-676 (1). Taq DNA polymerase (Qiagen, West Sussex, UK) with Q-Solution was used to amplify the 304 bp product. The cycling conditions were an initial denaturation at $95^{\circ} \mathrm{C}$ for $4 \mathrm{~min}$, followed by 35 cycles at $95^{\circ} \mathrm{C}$ for $1 \mathrm{~min}$, at $55^{\circ} \mathrm{C}$ for $1 \mathrm{~min}$ and at $72^{\circ} \mathrm{C}$ for $1.5 \mathrm{~min}$. The reaction vol- 
ume was $20 \mu \mathrm{L}$, and the primer concentration was $0.2 \mu \mathrm{M}$. The samples were then digested overnight with $10 \mathrm{U}$ of $B s t \mathrm{~N} 1$, which digests allele 2 exclusively into 160 and $144 \mathrm{bp}$ fragments. All pools were PCR amplified in triplicate.

The products were run on a $3 \%$ agarose gel. The loading buffer used was $30 \%$ glycerol plus acridine orange. Bromophenol blue and xylene cyanol were not used as they interfered with the quantification of bands. Acridine orange runs at approximately $50 \mathrm{bp}$. We used a 100 bp ladder (Promega, Madison, WI, USA) to size products. Ethidum bromide was added to the agarose to a final concentration of 0.1 $\mu \mathrm{g} / \mathrm{mL}$. Post-staining and destaining were also tried but did not offer any advantage (results not shown). The average amount of digested PCR products loaded on the gel was approximately $400 \mathrm{ng}$ as estimated from bands on a $100 \mathrm{bp}$ size ladder (Promega). The gel images were captured to disk as TIFF files for further analysis.

\section{Taqman Assay: $2041 \mathrm{G} \rightarrow \mathrm{T}$}

We investigated a $\mathrm{G}$ (allele 1) to $\mathrm{T}$ (allele 2) substitution at position 2046 in the COLIA1 gene. It can be reliably detected using fluorescent technology as well as enzymatic digestion of PCR products. Primers 2041-F: 5'-GTT
GTC TAG GTG CTG GAG GTT AGG-3' and 2041-R: 5'-GGC GAG GGA GGA GAG AAG G-3' were used. The Taqman probes used were 2041TET: 5'-CCC GCC CaC ATT CCC TGG-3' and 2041-FAM: 5'-CCC GCC CcC ATT CCC TGG-3' (PE Biosystems). The assay had previously been optimized (conditions available on request from S.R.). The probes were added before PCR in an optical 96-well plate (PE Biosystems) and an allelicdiscrimination assay using the $\mathrm{ABI}$ 7200 endpoint Taqman machine. The fluorescent detection system relies on two allele-specific fluorogenic probes, which only fluoresce when they are bound by template (6). All pools were PCR amplified in triplicate. The raw data were exported to a Microsoft Excel spreadsheet for analysis.

\section{Analysis of Results}

RFLP Agarose Gel Densitometry. The TIFF files saved were analyzed with Scan Analysis v.2.56 (Biosoft, Cambridge, UK) using the manufacturer's protocol on a Macintosh PowerPC $4600 / 250$ with an unused lane selected as background. Allele frequencies were estimated from the percentage of the signal represented by each band. Manipulation and editing of data were carried out using Excel and statistical

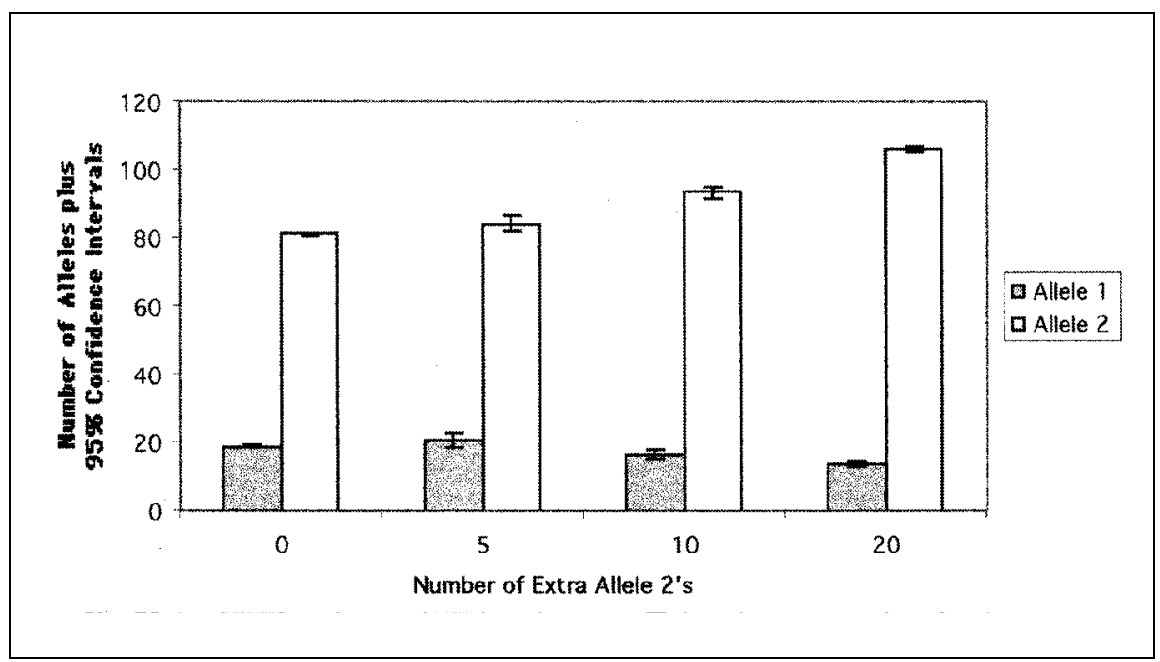

Figure 1. Results of DNA spiking experiment on the DRD2 -141 Del/Ins RFLP. Five, ten and twenty extra cutting alleles were added to a pool of 50 individuals. The error bars represent $95 \%$ confidence intervals. A difference of 10 alleles $(10 \%)$ is significant. The estimated frequency of allele 1 seemed to fall from 20 alleles to 13 as the amount of allele 2 rose. This is perhaps due to decreasing opportunities for heteroduplex formation as the ratio of allele 1 to allele 2 decreases. The allele frequency estimates were corrected using the equations derived from the calibration experiment illustrated in Figure 2. 


\section{Short Technical Reports}

analysis in SPSS ${ }^{\mathrm{TM}}$ (SPSS Science, Chicago, IL, USA).

\section{Taqman Assay Data}

Measurements for the two different dyes (probes) were corrected using the average ratio of the two measurements from three replicates of a heterozygote. The allele frequencies were then calculated from the percentage of the total signal each probe represented.

\section{RESULTS AND DISCUSSION}

The accuracy of DNA pooling is the degree to which estimated allele frequencies from pools correlate with the real frequencies. The sensitivity of DNA pooling in this context is the minimum difference detectable, in terms of the number of alleles present. In the case of microsatellites, the accuracy of the allele frequency estimates is low, perhaps because of stutter and differential amplification, but the sensitivity of the technique is high $(3,6)$. However, given the less complex nature of SNPs, it should be possible to achieve much greater accuracy combined with the same or better sensitivity. We looked at two methods by which this might be done.

\section{Densitometric Analysis of RFLPs}

We hypothesized that using conventional RFLP analysis, it should be possible, if done carefully, to estimate both allele frequencies and allele frequency differences in DNA pools. We examined the $-141 \mathrm{C}$ Del/Ins polymorphism in the DRD2 gene, which creates or disrupts a $B s t \mathrm{~N} 1$ restriction site. We performed PCR on a pool containing 20 allele 1's (C deletion) and 80 allele 2's (C insertion), digested the products and ran them on an agarose gel. The gel image was then saved to disk and the band intensities measured.

To test the sensitivity of the method to changes in allele frequencies, we also performed this analysis on pools containing 5, 10 and 20 extra allele 2's (Figure 1). While we found the method was sensitive to small changes ( 5 alleles) in allele frequency at $p<0.05$, it gave distorted estimates of the allele frequency with an overall $\mathrm{r}^{2}$ value of $0.96(r=$ correlation coefficient between two variables, $\mathrm{r}^{2}=$ measure of variation in one variable accounted for by the oth-

er variable). The allele frequency estimates were particularly distorted when one allele was $\leq 20 \%$. This is due to the formation of non-cutting heteroduplex

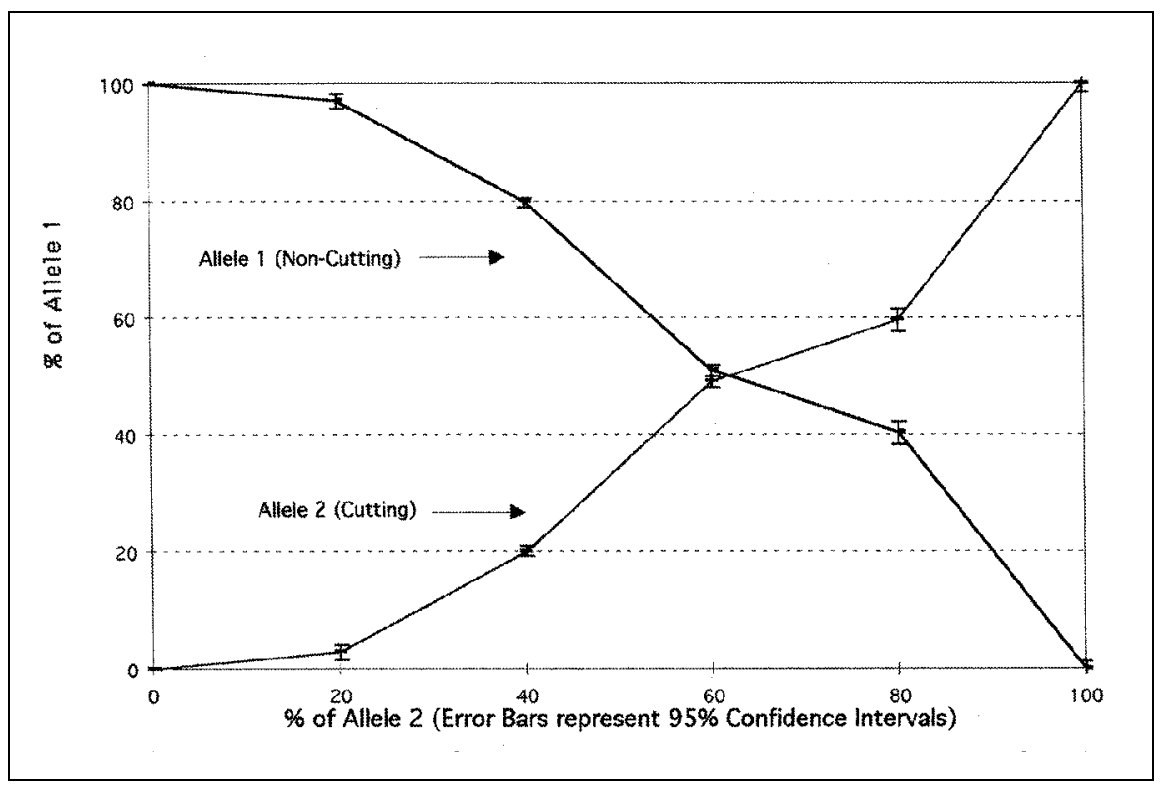

Figure 2. Calibration curve to correct for the distorted allele frequencies derived from pooling analysis of RFLPs. We constructed a calibration curve using pools containing different ratios of DNA from $1 / 1$ and $2 / 2$ homozygotes. Cubic regression equations were calculated: $y=0.000087 x^{3}-0.0186 x^{2}+$ $1.9306 x+6.9185$ (Allele 2) and $y=0.000087 x^{3}-0.0074 x^{2}+0.8057 x-0.3984$ (Allele 1). These equations were used to correct the allele frequency estimates obtained in other experiments (see Figure 1).

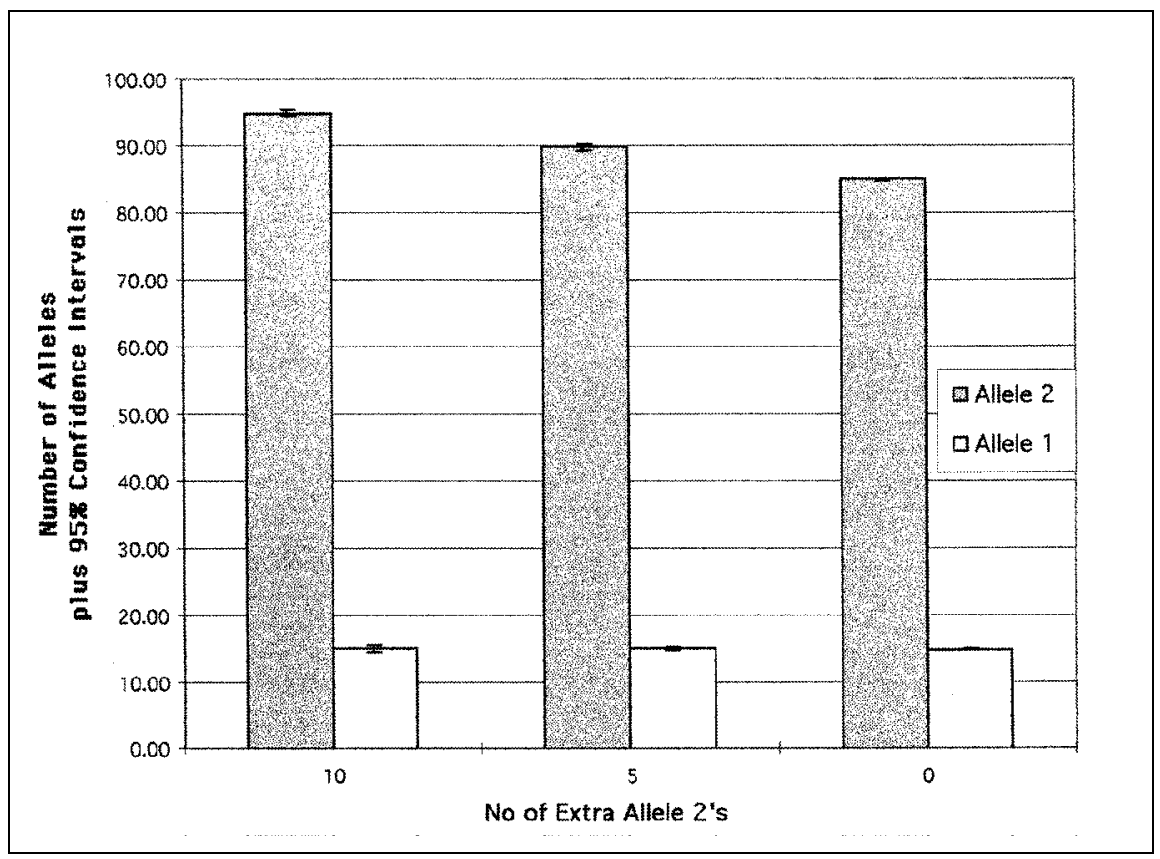

Figure 3. Graph showing accuracy and sensitivity of DNA pooling by Taqman. A pool of 50 individuals was analyzed, the amounts of DNA equivalent to 5 and 10 extra allele 2's were added to aliquots of this pool and the allele frequencies were calculated. The frequency estimates derived are almost identical to the expected values (see text). 


\section{Short Technical Reports}

molecules during PCR, during which the amount formed varies depending on the ratio of allele 1 to allele 2 . To correct for this, we performed a calibration experiment where pools containing different mixtures of the two alleles (0:100, 20:80, 40:60...) were analyzed.

The shape of the graph obtained (Figure 2) is roughly cubic, so cubic regression equations were calculated. These allowed us to correct the values of the estimates from the spiking experiment back to an approximation of their actual values (Figure 2). After correction, the overall accuracy of the technique as measured by $\mathrm{r}^{2}$ improved from 0.96 to 0.995 . More importantly, the allele frequency estimates, when one allele was $\leq 20 \%$, more closely approach the real values when these equations are applied. We conclude that this method could be used cheaply and reproducibly for DNA pooling experiments.

\section{Allele-Specific Fluorescent Probes}

Using the Taqman assay, genotypes are assigned based upon the ratio of fluorescence of the different probes. From this we hypothesised that in pooled samples, the proportion of each allele present should be reflected in the ratio of fluorescent signals observed. To test our hypothesis, we genotyped 50 individuals for the COL1A1 $2041 \mathrm{G} \rightarrow \mathrm{T}$ polymorphism on the ABI 7200 Taqman machine (PE Biosystems). DNA pools of these samples were then amplified three times and the pooled samples read on the ABI 7200 for comparison with their actual values. The results were highly consistent and reproducible.

Using a corrected unit fluorescence to compensate for differing levels of fluorescence from each probe, the average results for three PCRs of a pool containing 85 copies of allele 2 (2's) and 15 copies of allele 1 (1's) were obtained. An average of 85.17 for the 2's and 14.83 for the 1 's $(\mathrm{SD}=0.12$ for both) were estimated. To confirm the accuracy of the method, we spiked the original pool containing the 852 's and the 15 1's with 5 or 10 extra 2's (totals of 90 and 95). The mean of three replicates gave estimates of 89.89 for the 2's and 15.11 for the 1's in the pool containing 902 's and 151 's, and 95.002 's and 15.001 's in the pool containing 95 2's and 15 1's (Figure 3). When five alleles were added, the difference in measurements was greater than 5 standard deviations in all cases, which is equivalent to $P<0.0001$.

The allele frequencies are not distorted by pooling the DNA, and it seems the only limiting factor on accuracy in this system is the variation introduced when the pools are constructed and the DNA concentration 
measurements made. Therefore, this method holds great promise for the use of single base-pair polymorphisms in a scan of the entire human genome for genetic association with a DNA pooling methodology, although the expenses involved are considerably greater than those for RFLPs.

\section{CONCLUSION}

Two methods of DNA pooling analysis of SNPs are presented. The RFLP method is less accurate than the Taqman method but should still be sensitive enough for the rapid screening of candidate polymorphisms. With the Taqman method, the allele estimates are extremely accurate but the set-up costs per polymorphism are large (approximately $\$ 800$ ) and the optimization of the assay may present difficulties. On the other hand, the cost of genotyping samples individually by any method far exceeds this cost. As the Taqman technology becomes more developed, it will probably become a more viable technology for large-scale screening of many polymorphisms.

\section{REFERENCES}

1.Arinami, T., M. Gao, H. Hamaguchi and M. Toru. 1997. A functional polymorphism in the promoter region of the dopamine D2 receptor gene is associated with schizophrenia. Hum. Mol. Genet. 6:577-582.

2.Barcellos, L.F., W. Klitz, L. Field, R. Tobias, A.M. Bowcock, R. Wilson, M.P. Nelson et al. 1997. Association mapping of disease loci by use of a pooled DNA genomic screen. Am. J. Hum. Genet. 61:734-747.

3.Breen, G., P. Sham, T. Li, D. Shaw, D. Collier and D. St. Clair. Accuracy and sensitivity of DNA pooling with microsatellite repeats using capillary electrophoresis. Mol. Cell Probes (In press).

4.Daniels, J.K., N.M. Williams, R. Plomin, P. McGuffin and M.J. Owen. 1995. Selective DNA pooling using automated technology: a rapid technique for allelic association studies in quantitative traits and complex diseases. Psychiatr. Genet. Suppl. 5:S39.

5.Grant, S.F.A., D.M. Reid, G. Blake et al. 1996. Reduced bone density and osteoporosis associated with a polymorphic Sp1 binding site in the collagen type I alpha 1 gene. Nat. Genet. 14:203-205.

6.Lee, L.G., C.R. Connell and W. Bloch. 1993. Allele discrimination by nick translation PCR with fluorogenic probes. Nucleic Acids Res. 21:3761-3766.
7.Risch, N. and K. Merikangas. 1996 The future of genetic studies of complex human diseases. Science 273:1516-1517.

8.Zubenko, G.S., J.S. Stiffler, H.B. Hughes, M.R. Hurtt and B.P. Kaplan. 1998. Initial results of a genome survey for novel Alzheimer's disease risk genes: association with a locus on the $\mathrm{X}$ chromosome. Am. J. Med. Genet. 81:196-205.

Address correspondence to Dr. Gerome Breen, Dept. of Mental Health, University Medical Buildings, Foresterhill, Aberdeen, Scotland, AB25 2ZD, UK. Internet: gbreen@abdn.ac.uk

Received 19 July 1999; accepted 14 October 1999.

\section{G. Breen, D. Harold, S. Ralston, D. Shaw and D. St. Clair University of Aberdeen Aberdeen, Scotland, UK}

\section{Use of Fluorescent Microspheres to Localize In Vivo Gene Transfer Injection Sites}

BioTechniques 28:470-476 (March 2000)

\section{ABSTRACT}

The potential for using gene therapy to treat a variety of disease states is growing rapidly. Many vector types and delivery systems have been developed that allow the optimization of protein production levels and kinetics for a given therapeutic gene product. In cases in which a transient, localized delivery of gene product is desired, any determination of the locale of transfected tissue by non-marker genes is problematic. We describe a technique by which the use of fluorescent microspheres can help in identifying potentially transfected tissue. Adenovirus containing the gene for $\beta$-galactosidase ( $\beta$-gal) was mixed with fluorescent microspheres and injected into rat skeletal muscle and porcine myocardium. The injection sites could be visualized under ultraviolet light and correlated with $\beta$-gal enzyme expression. This method is simple, inexpensive and generally useful for in vivo gene transfer experiments.

\section{INTRODUCTION}

A variety of gene-therapy based treatments have demonstrated efficacy in animal models $(1,2,5-8,10-12)$ and several have entered or approach clinical trial status $(3,4,9)$. Among other things, the research preceding these studies requires the determination of gene product expression levels, expression kinetics and protein localization in transfected tissues. When the gene product cannot be detected visually, its localization can be difficult because a relatively small piece from a large tissue mass is often involved. One approach to this problem is to place sutures at the site of transgene administration, although this is impractical when the treated area is remotely located in the body (e.g., catheter-mediated administration). An alternative method would be the co-injection of a marker 